Pacific Journal of Mathematics

ON COVERING OF REAL LINE BY NULL SETS 


\title{
ON COVERING OF REAL LINE BY NULL SETS
}

\author{
TOMEK BARTOSZYNSKI
}

In this note it is proved that the least cardinal $\kappa$ such that $\mathbf{R}$ cannot be covered by $\kappa$ many null sets cannot have countable cofinality, provided $2^{\omega}$-scale exists and $2^{\omega}$ is regular cardinal. Using the same assumption a combinatorial characterisation of this cardinal is also found.

Let $\kappa_{m}$ be the least cardinal $\kappa$ such that the real line can be covered by $\kappa$ many null sets.

The goal of this paper is to give a combinatorial description of $\kappa_{m}$. This problem was stated by Arnold Miller in [Mi 1]. We also aim to find a solution to the question asked by David Fremlin whether $c f\left(\kappa_{m}\right)$ can be equal $\omega$.

We are able to answer the above questions using the additional assumption of the existence of $2^{\omega}$-scale in $\omega^{\omega}$.

It is well known that the cardinal $\kappa_{m}$ is the same in $\mathbf{R}$, as in $2^{\omega}$ or any uncountable Polish space with totally $\boldsymbol{\sigma}$-finite continuous measure. Thus without loss of generality we can work in the space $2^{\omega}$ with the Lebesgue measure.

We shall use standard notation. $\omega$ denotes the set of natural numbers. For $n, k<\omega$ the interval $[n, k)$ is the set $\{i<\omega: n \leq i<k\}$. For $n<\omega$ $2^{n}\left(2^{\omega}\right)$ is either the set of all 0-1 sequences of length $n(\omega)$ or the cardinal $2^{n}\left(2^{\omega}\right)$-depending on the context. For any set $X|X|$ denotes the cardinality of $X$. For any two finite sequences $s, t s \wedge t$ denotes concatenation of them. Finally, quantifiers $\forall^{\infty}$ and $\exists^{\infty}$ abbreviate "for all except finitely many" and "there exist infinitely many" respectively.

1. In this first section we introduce the notation of small sets and we prove basic properties of these sets.

We start with the following well-known lemma.

Lemma 1.1. Suppose $H \subseteq 2^{\omega}$ is a null set. Then there exists a sequence $J_{n} \subseteq 2^{n}$ for $n<\omega$ such that $\sum_{n=1}^{\infty}\left|J_{n}\right| 2^{-n}<\infty$ and $H \subseteq\left\{x \in 2^{\omega}: \exists^{\infty} n\right.$ $\left.x \uparrow n \in J_{n}\right\}$.

For the proof of Lemma 1.1 see [0]. 
Lemma 1.1 gives us some combinatorial representation of null sets. Nevertheless it turns out that this representation is not working satisfactorily. Hence we define a new family of sets which looks much more promising.

Definition. A set $H \subseteq 2^{\omega}$ is called small iff there exists a partition of $\omega$ into pairwise disjoint intervals $\left\{I_{n}: n<\omega\right\}$ and a sequence $\left\{J_{n}\right.$ : $n<\omega\}$ such that

(i) $J_{n} \subseteq 2^{I_{n}}$

(ii) $\sum_{n=1}^{\infty}\left|J_{n}\right| 2^{-\left|I_{n}\right|}$

(iii) $H \subseteq\left\{x \in 2^{\omega}: \exists^{\infty} n x \uparrow I_{n} \in J_{n}\right\}$.

Denote the set defined by sequences $\left\{I_{n}: n<\omega\right\}$ and $\left\{J_{n}: n<\omega\right\}$ by $\left(I_{n}, J_{n}\right)_{n=1}^{\infty}$.

Observe that condition (ii) guarantees that the set $\left(I_{n}, J_{n}\right)_{n=1}^{\infty}$ is null. The next lemma describes the relationship between null and small sets.

LEMMA 1.2. Every null set is a union of two small sets.

Proof. Suppose $H \subseteq 2^{\omega}$ is a null set. By Lemma 1.1 we can assume that $H=\left\{x \in 2^{\omega}: \exists^{\infty} n x \uparrow n \in J_{n}\right\}$ for some sequence $\left\{J_{n}: n<\omega\right\}$.

Fix some sequence of positive reals $\left\{\varepsilon_{n}: n<\omega\right\}$ such that $\sum_{n=1}^{\infty} \varepsilon_{n}<$ $\infty$.

Define sequences $\left\{n_{k}, m_{k}: k<\omega\right\}$ as follows

$$
\begin{aligned}
n_{0} & =0 \\
m_{k+1} & =\min \left\{u<\omega: 2^{n_{k}} \sum_{i=u}^{\infty}\left|J_{i}\right| 2^{-i}<\varepsilon_{k}\right\} \\
n_{k+1} & =\min \left\{u<\omega: 2^{m_{k+1}} \sum_{i=u}^{\infty}\left|J_{i}\right| 2^{-\imath}<\varepsilon_{k}\right\} .
\end{aligned}
$$

Now define

$$
\begin{aligned}
& I_{k}=\left[n_{k}, n_{k+1}\right), \\
& I_{k}^{\prime}=\left[m_{k}, m_{k+1}\right) \text { for } k<\omega .
\end{aligned}
$$

Let

$$
s \in F_{k} \quad \text { iff } s \in 2^{I_{k}} \& \exists i \in\left[m_{k+1}, n_{k+1}\right) \exists t \in J_{1}
$$$$
s \uparrow \operatorname{dom}(t) \cap \operatorname{dom}(s)=t \uparrow \operatorname{dom}(t) \cap \operatorname{dom}(s),
$$ 
and similarly

$$
\begin{aligned}
s \in F_{k}^{\prime} \quad \text { iff } s & \in 2^{I_{k}^{\prime}} \& \exists i \in\left[n_{k}, m_{k+1}\right) \exists t \in J_{i} \\
s & \uparrow \operatorname{dom}(t) \cap \operatorname{dom}(s)=t \uparrow \operatorname{dom}(t) \cap \operatorname{dom}(s) .
\end{aligned}
$$

From the above definitions easily follows that the sets $\left(I_{k}, F_{k}\right)_{k=1}^{\infty}$ and $\left(I_{k}^{\prime}, F_{k}^{\prime}\right)_{k=1}^{\infty}$ are small. Simply notice that $\left|F_{k}\right| / 2^{\left|I_{k}\right|}<\varepsilon_{k}$. Now we show that

$$
H \subseteq\left(I_{k}, F_{k}\right)_{k=1}^{\infty} \cup\left(I_{k}^{\prime}, F_{k}^{\prime}\right)_{k=1}^{\infty} .
$$

Suppose that $x \in H$. By our assumption it means that the set

$$
X=\left\{n<\omega: x \uparrow n \in J_{n}\right\} \text { is infinite. }
$$

Thus one of the following two sets is infinite

$$
X \cap \bigcup_{k=1}^{\infty}\left[m_{k+1}, n_{k+1}\right) \text { or } X \cap \bigcup_{k=1}^{\infty}\left[n_{k}, m_{k+1}\right) .
$$

It is just routine to check that if the frist of these sets is infinite then $x \in\left(I_{k}, F_{k}\right)_{k=1}^{\infty}$ and $x \in\left(I_{k}^{\prime}, F_{k}^{\prime}\right)_{k=1}^{\infty}$ if the second set is infinite.

The next lemma justifies the name given to our sets.

LEMMA 1.3. There exists a null set which is not small.

Proof. Let

$I_{n}=\left[2^{n}, 2^{n+1}\right)$,

$I_{n}^{\prime}=\left[2^{n}+2^{n-1}, 2^{n+1}+2^{n}\right)$ for $n<\omega$ and

$J_{n}=\left\{s \in 2^{I_{n}}: s\left(\max \left(I_{n}\right)\right)=s\left(\min \left(I_{n}\right)\right) \& s\left(\max \left(I_{n}\right)-1\right)\right.$

$$
\left.=s\left(\min \left(I_{n}\right)+1\right) \& \cdots \& s\left(\max \left(I_{n}\right)-n\right)=s\left(\min \left(I_{n}\right)+n\right)\right\},
$$

$J_{n}^{\prime}=\left\{s \in 2^{I_{n}^{\prime}}: s\left(\max \left(I_{n}^{\prime}\right)\right)=s\left(\min \left(I_{n}^{\prime}\right)\right) \& s\left(\max \left(I_{n}^{\prime}\right)-1\right)\right.$

$$
\left.=s\left(\min \left(I_{n}^{\prime}\right)+1\right) \& \cdots \& s\left(\max \left(I_{n}^{\prime}\right)-n\right)=s\left(\min \left(I_{n}^{\prime}\right)+n\right)\right\}
$$

for $n<\omega$.

It is easy to verify that the sets $\left(I_{n}, J_{n}\right)_{n=1}^{\infty}$ and $\left(I_{n}^{\prime}, J_{n}^{\prime}\right)_{n=1}^{\infty}$ are small.

We will show that the union of these two sets is not small. We will need the following technical lemma. 
LEMMA 1.4. Suppose that $\left(I_{n}^{0}, J_{n}^{0}\right)_{n=1}^{\infty} \subseteq\left(I_{n}^{1}, J_{n}^{1}\right)_{n=1}^{\infty}$ and let $F_{n}=\{k$ : $\left.I_{n}^{0} \cap I_{n}^{1} \neq 0\right\}$ for $n<\omega$.

Then for all except finitely many $n<\omega$ we have if $\operatorname{dom}(s)=\bigcup_{k \in F_{n}} I_{k}^{1}$ and $s \uparrow I_{n}^{0} \in J_{n}^{0}$ then there exists $k \in F_{n}$ and $t \in J_{k}^{1}$ such that $s \uparrow \operatorname{dom}(t)$ $=t$.

Proof. Suppose that this condition does not hold for some small sets $\left(I_{n}^{0}, J_{n}^{0}\right)_{n=1}^{\infty}$ and $\left(I_{n}^{1}, J_{n}^{1}\right)_{n=1}^{\infty}$.

It means that there exists an infinite set $X^{\prime} \subseteq \omega$ such that for $n \in X^{\prime}$ there exist $s_{n}$ with $\operatorname{dom}\left(s_{n}\right)=\cup_{k \in F_{n}} I_{k}^{1}$ such that $s_{n} \uparrow I_{n}^{0} \in J_{n}^{0}$ and for every $k \in F_{n}$ and for every $t \in J_{k}^{1} s_{n} \uparrow \operatorname{dom}(t) \neq t$.

Let $X$ be an infinite subset of $X^{\prime}$ such that for $n, k \in X F_{n} \cap F_{k}=0$. Now define $x \in 2^{\omega}$ as follows

$$
x \uparrow I_{k}^{1}=\left\{\begin{array}{l}
s_{n} \uparrow I_{k}^{1} \quad \text { if } k \in F_{n} \& n \in X \\
\text { otherwise any element not belonging to } J_{k}^{1} .
\end{array}\right.
$$

It is easy to see that $x \in\left(I_{n}^{0}, J_{n}^{0}\right)_{n=1}^{\infty}$ and $x \notin\left(I_{n}^{1}, J_{n}^{1}\right)_{n=1}^{\infty}$ which is a contradiction.

Now suppose that $\left(I_{n}, J_{n}\right)_{n=1}^{\infty} \cup\left(I_{n}^{\prime}, J_{n}^{\prime}\right)_{n=1}^{\infty}$ is contained in the set $\left(I_{n}^{\prime \prime}, J_{n}^{\prime \prime}\right)_{n=1}^{\infty}$.

Define $n_{k}=\max \left(I_{k}^{\prime \prime}\right)$ for $k<\omega$.

Without loss of generality we can assume that each interval $I_{k}^{\prime \prime}$ intersects at least two $I_{n}$ 's and $I_{n}^{\prime}$ 's.

Since the distances between the endpoints of $I_{n}$ and $I_{n}^{\prime}$ are getting bigger and bigger either there are infinitely many intervals $I_{k}$ such that each $I_{k}$ contains an element $n_{l}$ for some $l<\omega$ and the distance of $n_{l}$ from the endpoints of $I_{k}$ is greater than $k$ or the sequence $I_{k}^{\prime}$ has this property.

Without loss of generality we can assume that the first case is satisfied.

Fix sufficiently large $k<\omega$ such that $I_{k}$ has the above property.

Assume that $I_{k} \subseteq I_{l}^{\prime \prime} \cup I_{l+1}^{\prime \prime}$.

Notice that from Lemma 1.4 follows that

$$
\begin{aligned}
\left\{s \in 2^{I_{l}^{\prime \prime} \cup I_{l+1}^{\prime \prime}}: s \uparrow I_{k} \in J_{k}\right\} \subseteq & \left\{s \in 2^{I_{l}^{I^{\prime}} \cup I_{l+1}^{\prime \prime}}: s \uparrow I_{l}^{\prime \prime} \in J_{l}^{\prime \prime}\right\} \\
& \cup\left\{s \in 2^{I_{l}^{\prime \prime} \cup I_{l+1}^{\prime \prime}}: s \uparrow I_{l+1}^{\prime \prime} \in J_{l+1}^{\prime \prime}\right\} .
\end{aligned}
$$


We will show that in this case sets $J_{l}^{\prime \prime}$ and $J_{l+1}^{\prime \prime}$ are large, i.e. that

$$
\left|J_{l}^{\prime \prime}\right| 2^{-\left|I_{l}^{\prime \prime}\right|}+\left|J_{l+1}^{\prime \prime}\right| 2^{-\left|I_{l+1}^{\prime \prime}\right|} \geq \frac{1}{2} \text {. }
$$

This will finish the proof of Lemma 1.3 because then $\sum_{n=1}^{\infty}\left|J_{l}^{\prime \prime}\right| 2^{-\left|I_{i}^{\prime \prime}\right|}=\infty$, since $(*)$ holds for infinitely many $l$ 's. Thus it is enough to show that

$$
\begin{aligned}
& \left|\left\{s \in 2^{I_{l}^{\prime \prime} \cup I_{l+1}^{\prime \prime}}: s \uparrow I_{l}^{\prime \prime} \in J_{l}^{\prime \prime}\right\} \cup\left\{s \in 2^{I_{l}^{\prime \prime} \cup I_{l+1}^{\prime \prime}}: s \uparrow I_{l+1}^{\prime \prime} \in J_{l+1}^{\prime \prime}\right\}\right| \\
& \quad \geq 2^{\left|I_{l}^{\prime \prime} \cup I_{l+1}^{\prime \prime}\right|-1} .
\end{aligned}
$$

Recall that

$$
\begin{aligned}
J_{k}=\left\{s \in 2^{I_{k}}\right. & : s\left(\max \left(I_{k}\right)\right)=s\left(\min \left(I_{k}\right)\right) \\
& \left.\& \cdots \& s\left(\max \left(I_{k}\right)-k\right)=s\left(\min \left(I_{k}\right)+k\right)\right\} \quad \text { for } k<\omega .
\end{aligned}
$$

For $s \in 2^{I_{i}^{\prime \prime} \cup I_{i+1}^{\prime \prime}}$ define $s^{*} \in 2^{I_{i}^{\prime \prime} \cup I_{i+1}^{\prime \prime}}$ as follows

$$
s^{*}(n)= \begin{cases}s\left(\max \left(I_{k}\right)-i\right) & \text { if } n=\min \left(I_{k}\right)+i, i \leq k, \\ s\left(\min \left(I_{k}\right)+i\right) & \text { if } n=\max \left(I_{k}\right)-i, i \leq k \\ s(n) & \text { in other cases. }\end{cases}
$$

Notice that $s=s^{*}$ iff $s \uparrow I_{k} \in J_{k}$.

Claim. For any $s \in 2^{I_{i}^{\prime \prime} \cup I_{i+1}^{\prime \prime}}$ either $s$ or $s^{*}$ belongs to

$$
\left\{s \in 2^{I_{l}^{\prime \prime} \cup I_{l+1}^{\prime \prime}}: s \uparrow I_{l}^{\prime \prime} \in J_{l}^{\prime \prime}\right\} \cup\left\{s \in 2^{I_{l}^{\prime \prime} \cup I_{l+1}^{\prime \prime}}: s \uparrow I_{l+1}^{\prime \prime} \in J_{l+1}^{\prime \prime}\right\} \text {. }
$$

Proof of the Claim. Fix $s \in 2^{I_{l}^{\prime \prime} \cup I_{l+1}^{\prime \prime}}$ and define $t=s \uparrow I_{l}^{\prime \prime} \wedge s^{*} \uparrow I_{l+1}$. Notice that $t=t^{*}$. Hence

$$
t \in\left\{s \in 2^{I_{l}^{\prime \prime} \cup I_{l+1}^{\prime \prime}}: s \uparrow I_{l}^{\prime \prime} \in J_{l}^{\prime \prime}\right\} \cup\left\{s \in 2^{I_{l}^{\prime \prime} \cup I_{l+1}^{\prime \prime}}: s \uparrow I_{l+1}^{\prime \prime} \in J_{l+1}^{\prime \prime}\right\} .
$$

By the definition of $t$ it means that either $s$ or $s^{*}$ belongs to this set.

By previous remarks this finishes the proof of Lemma 1.3.

We will conclude this section with the following lemma.

Lemma 1.5. Suppose that $H \subseteq 2^{\omega}$ can be covered by some null set of type $F_{\sigma}$. Then $H$ is small.

We leave an easy proof to the reader.

2. Covering of $\mathbf{R}$ by null sets. Let us start with some definitions. Suppose $f \in \omega^{\omega}$ and $\sum_{n=1}^{\infty} f(n)^{-1}<\infty$. 
Then define

$$
\begin{aligned}
\varphi \in \Sigma_{f} & \text { iff } \varphi \in\left([\omega]^{<\omega}\right)^{\omega} \& \varphi(n) \subseteq f(n) \text { for } n<\omega \\
& \& \sum_{n=1}^{\infty}|\varphi(n)| f(n)^{-1}<\infty,
\end{aligned}
$$

and let

$$
X_{f}=\prod_{n=1}^{\infty}\{i \in \omega: i \leq f(n)\} .
$$

Let $\Sigma$ and $X$ denote $\Sigma_{f}$ and $X_{f}$ respectively for $f(n)=2^{n}$ for $n<\omega$. For $f \in \omega^{\omega}$ let $\mathbf{T}_{f}$ denote the following sentence

$$
\forall \Phi \subseteq_{|\Phi|<2^{\omega}} \Sigma_{f} \exists h \in X_{f} \forall \varphi \in \Phi \forall^{\infty} n h(n) \notin \varphi(n) .
$$

The proof of the following lemma is left to the reader.

LEMMA 2.1. For any functions $f, g \in \omega^{\omega}$ such that $\sum_{n=1}^{\infty} f(n)^{-1}<\infty$ and $\sum_{n=1}^{\infty} g(n)^{-1}<\infty$ we have

$$
\mathbf{T}_{f} \equiv \mathbf{T}_{g}
$$

Before stating the main result of this section recall that $\mathbf{B}(m)$ denotes the sentence saying that $\mathbf{R}$ is not the union of less than $2^{\omega}$ null sets.

Let $\mathbf{D}$ denote the following sentence

$$
\forall F \subseteq_{|F|<2^{\omega}} \omega^{\omega} \exists g \in \omega^{\omega} \forall f \in F \forall n f(n)<g(n) .
$$

Observe that if $2^{\omega}$ is a regular cardinal then $\mathbf{D}$ is equivalent to the existence of $2^{\omega}$-scale in $\omega^{\omega}$.

TheOrem 2.2. Assume D. Then

$$
\mathbf{B}(m) \equiv \forall \Phi \subseteq_{|\Phi|<2^{\omega}} \Sigma \exists h \in X \exists \varphi \in \Phi \forall^{\infty} n h(n) \notin \varphi(n) .
$$

Proof. $\Rightarrow$ This implication is very easy since any element $\varphi \in \Sigma$ corresponds with the null subset of $2^{\omega}$ : since $\varphi(n) \subseteq 2^{n}$ we can identify $\varphi(n)$ with a certain subset of $0-1$ sequences of length $n$. Now consider the set $H_{\varphi}=([1+2+\cdots+(n-1), 1+2+\cdots+n), \varphi(n))_{n=1}^{\infty}$. For given family $\Phi \subseteq \Sigma$ apply $\mathbf{B}(m)$ to the family $\left\{H_{\varphi}: \varphi \in \Phi\right\}$ to obtain a required function $h \in X$.

$\Leftarrow$ Suppose $\left\{H_{\alpha}: \alpha<\theta<2^{\omega}\right\}$ is a family of null sets. By Lemma 1.1 we can assume that for $\alpha<\theta$

$$
H_{\alpha}=\left\{x \in 2^{\omega}: \exists^{\infty} n x \uparrow n \in \underline{J}_{n}^{\alpha}\right\} \text { for some sequence }\left\{\underline{J}_{n}^{\alpha}: n<\omega\right\}
$$
such that $\sum_{n=1}^{\infty}\left|\underline{J}_{n}^{\alpha}\right| 2^{-n}<\infty$. 
Claim. There exists a family $\left\{\left\{J_{k}^{\alpha}: k<\omega\right\}: \alpha<\theta\right\}$ and a sequence of natural numbers $\left\{u_{k}: k<\omega\right\}$ such that

(i) $\forall \alpha \forall k J_{k}^{\alpha} \subseteq 2^{u_{k}}$,

(ii) $H_{\alpha} \subseteq\left\{x \in 2^{\omega}: \exists^{\infty} k x \uparrow u_{k} \in J_{k}^{\alpha}\right\}$ for $\alpha<\theta$,

(iii) $\forall \alpha \forall k\left|J_{k}^{\alpha}\right| 2^{-u_{k}}<2^{-k}$.

Proof. For $\alpha<\theta$ and $k<\omega$ define

$$
n_{k}^{\alpha}=\min \left\{n: \sum_{i=n}^{\infty}\left|\underline{J}_{1}^{\alpha}\right| 2^{-\imath}<2^{-k}\right\} \text {. }
$$

Using $\mathbf{D}$ we can find an increasing sequence $\left\{u_{k}: k<\omega\right\}$ such that

$$
\forall \alpha<\theta \quad \forall^{\infty} k n_{k}^{\alpha}<u_{k} \text {. }
$$

Define for $\alpha<\theta$ and $k<\omega$

$$
J_{k}^{\alpha}=\left\{s \in 2^{u_{k}}: \exists i \in\left(u_{k-1}, u_{k}\right] s \uparrow i \in \underline{J}_{i}^{\alpha}\right\} .
$$

It is easy to check that the above family satisfies conditions (i)-(iii).

Let

$$
H_{\alpha}^{\prime}=\left\{x \in 2^{\omega}: \exists^{\infty} k x \uparrow u_{k} \in J_{k}^{\alpha}\right\} \text { for } \alpha<\theta .
$$

Observe that $H_{\alpha} \subseteq H_{\alpha}^{\prime}$ for $\alpha<\theta$. Thus in order to finish the proof we have to find a real $z \in 2^{\omega}$ such that

$$
z \notin H_{\alpha}^{\prime} \text { for } \alpha<\theta .
$$

Fix any sequence of positive reals $\left\{\varepsilon_{n}: n<\omega\right\}$ such that $\sum_{n=1}^{\infty} 2^{n} \varepsilon_{n}<\infty$.

Using Lemma 1.2 we can express every set $H_{\alpha}^{\prime}$ as a union of two small sets. Moreover, condition (iii) guarantees that we can do it uniformly for all sets $H_{\alpha}^{\prime}$. To be more precise: we can find two sequences $\left\{n_{k}, m_{k}\right.$ : $k<\omega\}$ of natural numbers such that

$$
n_{k}<m_{k}<n_{k+1}<m_{k+1} \text { for } k<\omega .
$$

We can also find families $L_{k}^{\alpha}, \underline{L}_{k}^{\alpha}$ for $\alpha<\theta, k<\omega$ such that

(i) $L_{k}^{\alpha} \subseteq 2^{\left[n_{k}, n_{k+1}\right)}, \underline{L}_{k}^{\alpha} \subseteq 2^{\left[m_{k}, m_{k+1}\right)}$ for $\alpha<\theta, k<\omega$,

(ii) $H_{\alpha}^{\prime} \subseteq\left(\left[n_{k}, n_{k+1}\right), L_{k}^{\alpha}\right)_{k=1}^{\infty} \cup\left(\left[m_{k}, m_{k+1}\right), \underline{L}_{k}^{\alpha}\right)_{k=1}^{\infty}$,

(iii) $\left|L_{k}^{\alpha}\right| 2^{n_{k}-n_{k+1}}<\varepsilon_{k},\left|\underline{L}_{k}^{\alpha}\right| 2^{m_{k}-m_{k+1}}<\varepsilon_{k}$ for $\alpha<\theta$.

Denote

$$
I_{k}=\left[n_{k}, n_{k+1}\right) \text { and } \underline{I}_{k}=\left[m_{k}, m_{k+1}\right) \text { for } k<\omega .
$$

Notice that if we define $f(k)=2^{\left|I_{k}\right|}$ for $k<\omega$ then each set $\left(I_{k}, L_{k}^{\alpha}\right)_{k=1}^{\infty}$ can be coded as an element $\varphi_{\alpha} \in \Sigma_{f}$. Moreover if $x \in X_{f}$ and $\forall^{\infty} n x(n) \notin \varphi_{\alpha}(n)$ then we can decode from $x$ a real $\underline{x} \in 2^{\omega}$ such that $\underline{x} \notin\left(I_{k}, L_{k}^{\alpha}\right)_{k=1}^{\infty}$. 
Hence by Lemma 2.1 our assumption is equivalent to the fact that

$$
\begin{aligned}
& 2^{\omega}-\bigcup\left\{\left(I_{k}, L_{k}^{\alpha}\right)_{k=1}^{\infty}: \alpha<\theta\right\} \neq 0 \text { and } \\
& 2^{\omega}-\bigcup\left\{\left(\underline{I}_{k}, \underline{L}_{k}^{\alpha}\right)_{k=1}^{\infty}: \alpha<\theta\right\} \neq 0 .
\end{aligned}
$$

All we have to show is that the intersection of the above two sets is also nonempty.

Fix $k<\omega$ and consider intervals $I_{k}$ and $\underline{I}_{k}$.

Let

$S_{k}^{\alpha}=\left\{s \in 2^{\left[n_{k}, m_{k}\right)}: s\right.$ has at least $2^{n_{k+1}-m_{k}-k}$ extensions inside $\left.L_{k}^{\alpha}\right\}$ for $\alpha<\theta$.

We have to estimate how big $S_{k}^{\alpha}$ can be.

Clearly

$$
\left|S_{k}^{\alpha}\right| 2^{n_{k+1}-m_{k}-k} \leq\left|L_{k}^{\alpha}\right| \leq \varepsilon_{k} 2^{n_{k+1}-n_{k}}
$$

Hence

$$
\left|S_{k}^{\alpha}\right| \leq 2^{k} \varepsilon_{k} 2^{m_{k}-n_{k}} \quad \text { for } \alpha<\theta \text { and } k<\omega .
$$

Similarly if we define

$$
\underline{S}_{k}^{\alpha}=\left\{s \in 2^{\left[n_{k}, m_{k}\right)}: s \text { has at least } 2^{n_{k}-m_{k-1}-k} \text { extensions inside } L_{k-1}\right\}
$$

$$
\text { for } \alpha<\theta \text { and } k<\omega
$$

then

$$
\left|\underline{S}_{k}^{\alpha}\right| \leq 2^{k} \varepsilon_{k} 2^{m_{k}-n_{k}} \quad \text { for } \alpha<\theta .
$$

Now define new small sets $\left(I_{k}, R_{k}^{\alpha}\right)_{k=1}^{\infty}$ as follows: for $\alpha<\theta, k<\omega$

$$
s \in R_{k}^{\alpha} \quad \text { iff } s \uparrow\left[n_{k}, m_{k}\right) \in S_{k}^{\alpha} \cup \underline{S}_{k}^{\alpha} .
$$

By our previous remarks the sets $\left(I_{k}, R_{k}^{\alpha}\right)_{k=1}^{\infty}$ are small since $\sum_{k=1}^{\infty} 2^{k} \varepsilon_{k}$ $<\infty$ and $\left|R_{k}^{\alpha}\right| 2^{-\left|I_{k}\right|} \leq 2^{k-1} \varepsilon_{k}$ for every $\alpha<\theta$ and $k<\omega$. Therefore by our assumption and Lemma 2.1 we can find a real $x \in 2^{\omega}$ such that

$$
x \notin \bigcup\left\{\left(I_{k}, R_{k}^{\alpha}\right)_{k=1}^{\infty}: \alpha<\theta\right\} .
$$

Now for $\alpha<\theta$ define sets $\left(W_{k}, T_{k}^{\alpha}\right)_{k=1}^{\infty}$ as follows

$$
\begin{gathered}
W_{k}=\left[m_{k}, n_{k}\right) \quad \text { for } k<\omega . \\
T_{k}^{\alpha}=\left\{s \in 2^{\left[m_{k}, n_{k+1}\right)}: x \uparrow\left[n_{k}, m_{k}\right) \wedge s \in L_{k}^{\alpha}\right. \text { or } \\
\left.s \wedge x \uparrow\left[n_{k+1}, m_{k+1}\right) \in \underline{L}_{k}^{\alpha}\right\} \quad \text { for } \alpha<\theta \text { and } k<\omega .
\end{gathered}
$$


Notice that the definition of the sets $\left(I_{k}, R_{k}^{\alpha}\right)_{k=1}^{\infty}$ for $\alpha<\theta$ and the choice of $x$ guarantees that the sets $\left(W_{k}, T_{k}^{\alpha}\right)_{k=1}^{\infty}$ are small. In fact one can show that

$$
\forall \alpha<\theta \forall^{\infty} k\left|T_{k}^{\alpha}\right| 2^{-\left|W_{k}\right|} \leq 2^{1-k} .
$$

Using the assumption and Lemma 2.1 we can find a real $y \in 2^{\omega}$ such that

$$
y \notin \bigcup\left\{\left(W_{k}, T_{k}^{\alpha}\right)_{k=1}^{\infty}: \alpha<\theta\right\} .
$$

Define $z \in 2^{\omega}$ in the following way

$$
z(n)= \begin{cases}y(n) & \text { if } n \in \bigcup\left\{W_{k}: k<\omega\right\} \\ x(n) & \text { in other cases. }\end{cases}
$$

We will show that

$$
z \notin\left(I_{k}, L_{k}^{\alpha}\right)_{k=1}^{\infty} \cup\left(\underline{I}_{k}, \underline{L}_{k}^{\alpha}\right)_{k=1}^{\infty} \text { for } \alpha<\theta .
$$

Assume the opposite and suppose that $z \in\left(I_{k}, L_{k}^{\alpha}\right)_{k=1}^{\infty}$ for some $\alpha<\theta$. It means that for infinitely many $k<\omega z \uparrow I_{k} \in L_{k}^{\alpha}$. Now two cases are possible:

Case 1 . There are infinitely many $k$ such that $z \uparrow\left[n_{k}, m_{k}\right)$ has at least $2^{n_{k+1}-n_{k}-k}$ extensions inside $L_{k}^{\alpha}$. That means that $z \in\left(I_{k}, R_{k}^{\alpha}\right)_{k=1}^{\infty}$ and since $z \uparrow\left[n_{k}, m_{k}\right)=x \uparrow\left[n_{k}, m_{k}\right)$ for every $k$ we have that $x \in$ $\left(I_{k}, R_{k}^{\alpha}\right)_{k=1}^{\infty}$. Contradiction.

Case 2. For all except finitely many $k z \uparrow\left[n_{k}, m_{k}\right.$ ) has less than $2^{n_{k+1}-n_{k}-k}$ extensions inside $L_{k}^{\alpha}$.

In this case $z \in\left(W_{k}, T_{k}^{\alpha}\right)_{k=1}^{\infty}$ which is impossible by the choice of $y$. The similar reasoning shows that $z \notin\left(\underline{I}_{k}, \underline{L}_{k}^{\alpha}\right)_{k=1}^{\infty}$ for $\alpha<\theta$.

Recall that $\kappa_{m}$ is the least cardinal $\kappa$ such that $\mathbf{R}$ can be covered by $\kappa$ many null sets. Let $\kappa_{D}$ be the smallest cardinal $\kappa$ such that

$$
\forall F \subseteq_{|F|<\kappa} \omega^{\omega} \exists g \in \omega^{\omega} \forall f \in F \exists^{\infty} n g(n)<f(n) .
$$

Observe that Theorem 2.2 characterises $\kappa_{m}$ as well as $\mathbf{B}(m)$. I.e. assuming that $\kappa_{m}<\kappa_{D}, \kappa_{m}$ is the least cardinal $\kappa$ such that there exists a family $\Phi \subseteq \Sigma$ of size $\kappa$ such that

$$
\forall x \in X \exists \varphi \in \Phi \exists^{\infty} n x(n) \in \varphi(n) .
$$

Theorem 2.2 enables us to give a partial solution to a question of David Fremlin about cofinality of $\kappa_{m}$. 
THEOREM 2.3. If $\kappa_{m} \leq \kappa_{D}$ then $c f\left(\kappa_{m}\right)>\omega$.

Proof. Assume opposite. Let $\Phi \subseteq \Sigma$ be the least family such that

$$
\forall x \in X \exists \varphi \in \Phi \exists^{\infty} n x(n) \in \varphi(n) .
$$

Since $c f(|\Phi|)=\omega$ we can find an increasing sequence $\left\{\Phi_{n}: n<\omega\right\}$ such that $\bigcup\left\{\Phi_{n}: n<\omega\right\}=\Phi$ and $\left|\Phi_{n}\right|<|\Phi|$ for every $n<\omega$.

Using the assumptions that $\Phi$ has the least possible power we can find elements $x_{n} \in X$ for $n<\omega$ such that

$$
\forall_{n} \forall \varphi \in \Phi_{n} \forall^{\infty} k \quad x_{n}(k) \notin \varphi(k) .
$$

Now for $\varphi \in \Phi$ define functions $f_{\varphi} \in \omega^{\omega}$ as follows

$$
f_{\varphi}(n)=\left\{\begin{array}{l}
\min \left\{m: \forall k \geq m x_{n}(k) \notin \varphi(k)\right\} \\
0 \quad \text { if minimum does not exist. }
\end{array}\right.
$$

Using the fact that $\kappa_{m} \leq \kappa_{D}$ and that $c f\left(\kappa_{d}\right)>\omega$ we can find an increasing function $f \in \omega^{\omega}$ such that $f(0)=0$ and

$$
\forall \varphi \in \Phi \forall^{\infty} n f_{\varphi}(n)<f(n) \text {. }
$$

For $k<\omega$ define

$$
x(k)=x_{n}(k) \text { if } k \in[f(n), f(n+1)) .
$$

It is easy to see that

$$
\forall \varphi \in \Phi \forall^{\infty} n x(n) \notin \varphi(n) \quad \text { contradiction. }
$$

\section{Sets of cardinality $<2^{\omega}$.}

Theorem 3.1. Suppose that $X \subseteq 2^{\omega}$ and $|X|<2^{\omega}$. Then $X$ is null iff $X$ is small.

Proof. $\leftarrow$ Obvious.

$\rightarrow$ Suppose $X \subseteq 2^{\omega}$ and $X$ is contained in some null set $H$. By 1.2 we can decompose $H$ onto small sets $\left(I_{n}, J_{n}\right)_{n=1}^{\infty}$ and $\left(\underline{I}_{n}, \underline{J}_{n}\right)_{n=1}^{\infty}$. Without loss of generality we can assume that each interval $I_{n}$ intersects exactly two intervals $\underline{I}_{k}$.

For $x \in X$ define

$$
\operatorname{supp}(x)=\left\{n: x \uparrow I_{n} \in J_{n}\right\} .
$$


Since $|X|<2^{\omega}$ there exists an infinite set $Y \subseteq \omega$ such that for $x \in X$

$$
|\operatorname{supp}(x)-Y|=\omega \quad \text { whenever }|\operatorname{supp}(x)|=\omega .
$$

To find such a set consider a family of pairwise almost disjoint subsets of $\omega$ of cardinality $2^{\omega}$. One of the sets in this family has desired property.

Take

$$
H^{\prime}=\left(I_{n}, J_{n}\right)_{n \in Y} \cup\left(\underline{I}_{n}, \underline{J}_{n}\right)_{n=1}^{\infty} .
$$

It is not very hard to check that the set $H^{\prime}$ is small and that $X \subseteq H^{\prime}$.

We will conclude this section with the analogue of Theorem 2.2. Let SD denote the following sentence

$$
\exists F \subseteq_{|F|<2^{\omega}} \omega^{\omega} \forall g \in \omega^{\omega} \exists f \in F \forall^{\infty} n g(n)<f(n) .
$$

Let $\mathbf{U}(m)$ be the sentence saying that every set of cardinality less than $2^{\omega}$ is null. The following theorem is an easy corollary of Theorem 3.1.

TheOREM 3.2. Assume SD. Then

$$
\begin{aligned}
& \mathbf{U}(m) \equiv \exists g \in \omega^{\omega} \forall F \subseteq_{|F|<2^{\omega}} X_{g} \exists \varphi \in \Sigma_{g} \forall f \in F \\
& \forall^{\infty} n f(n) \in \varphi(n) \text {. }
\end{aligned}
$$

REMARKs. (1) It is well known that $\mathbf{B}(m)$ and $\mathbf{D}$ have little in common. Actually any of $\mathbf{B}(m) \& \neg \mathbf{D}, \mathbf{B}(m) \& \mathbf{D}, \neg \mathbf{B}(m) \& \mathbf{D}$ and $\neg \mathbf{B}(m) \& \neg \mathbf{D}$ is consistent with $\mathbf{Z F C}$.

(2) All problems considered in this paper have their analogues in the ideal of meager subsets of $\mathbf{R}$. In particular, the cardinal $\kappa_{B}$ defined as the cardinality of the least covering of the real line by meager sets, was studied by A. Miller (see [Mi 1]). He proved that $c f\left(\kappa_{B}\right)>\omega$. Sentences $\mathbf{B}(c)$ and $\mathbf{U}(c)$ analogous to $\mathbf{B}(m)$ and $\mathbf{U}(m)$ along with several other properties of the ideals of meager and null sets were studied in [Mi 2] and [Ba].

\section{REFERENCES}

[Ba] T. Bartoszynski, Combinatorial aspects of measure and category, to appear in Fund. Math.

[Mi 1] A. Miller, Baire category theorem and cardinals of countable cofinality, J. Symbolic Logic, 47 (1982). 
[Mi 2] A. Miller, Some properties of measure and category, Trans. Amer. Math. Soc., 266 (1981).

[O] J. Oxtoby, Measure and Category, Springer Verlag.

Received November 21, 1985 and in revised form February 4, 1987.

WARSAW UNIVERSITY

Warsaw, Poland

AND

UNIVERSITY OF CALIFORNIA

BERKELEY, CA 94720 


\section{PACIFIC JOURNAL OF MATHEMATICS EDITORS}

\author{
V. S. VARADARAJAN \\ (Managing Editor) \\ University of California \\ Los Angeles, CA 90024 \\ Herbert Clemens \\ University of Utah \\ Salt Lake City, UT 84112 \\ R. FINN \\ Stanford University \\ Stanford, CA 94305
}

ROBION KIRBY

University of California

Berkeley, CA 94720

C. C. MOORE

University of California

Berkeley, CA 94720

HAROLD STARK

University of California, San Diego

La Jolla, CA 92093

\section{ASSOCIATE EDITORS}
R. ARENS
E. F. BECKENBACH
B. H. NEUMANN
F. WOLF
K. YOSHIDA (1906-1982)

\section{SUPPORTING INSTITUTIONS}
UNIVERSITY OF ARIZONA
UNIVERSITY OF OREGON
UNIVERSITY OF BRITISH COLUMBIA UNIVERSITY OF SOUTHERN CALIFORNIA
CALIFORNIA INSTITUTE OF TECHNOLOGY
UNIVERSITY OF CALIFORNIA
STANFORD UNIVERSITY
MONTANA STATE UNIVERSITY
UNIVERSITY OF HAWAII
UNIVERSITY OF NEVADA, RENO
UNIVERSITY OF TOKYO
NEW MEXICO STATE UNIVERSITY
UNIVERSITY OF UTAH
OREGON STATE UNIVERSITY
WASHINGTON STATE UNIVERSITY UNIVERSITY OF WASHINGTON 


\section{Pacific Journal of Mathematics}

\section{Vol. 131, No. $1 \quad$ November, 1988}

Tomek Bartoszynski, On covering of real line by null sets $\ldots \ldots \ldots \ldots \ldots 1$

Allen Davis Bell and Kenneth R. Goodearl, Uniform rank over differential operator rings and Poincaré-Birkhoff-Witt extensions $\ldots \ldots \ldots \ldots \ldots 13$

Brian Boe, Thomas Jones Enright and Brad Shelton, Determination of the intertwining operators for holomorphically induced representations of Hermitian symmetric pairs

Robert F. Brown, Topological identification of multiple solutions to

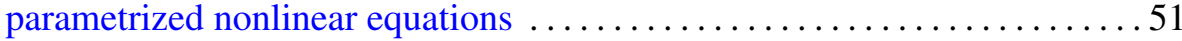

Marc R. M. Coppens, Weierstrass points with two prescribed nongaps ......71

Peter Larkin Duren and M. Schiffer, Grunsky inequalities for univalent functions with prescribed Hayman index .................... 105

Robert Greene and Hung-Hsi Wu, Lipschitz convergence of Riemannian manifolds

Kathryn E. Hare, Arithmetic properties of thin sets

Neal I. Koblitz, Primality of the number of points on an elliptic curve over a

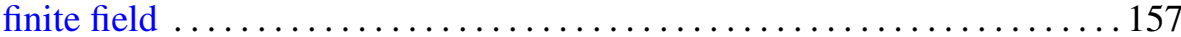

Isabel Dotti de Miatello and Roberto Jorge Miatello, Transitive isometry groups with noncompact isotropy

Raymond A Ryan, Weakly compact holomorphic mappings on Banach

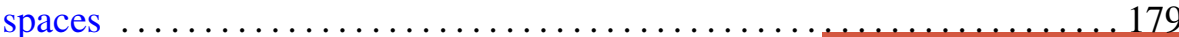

Tudor Zamfirescu, Curvature properties of typical convex surfaces 